\title{
Intangible Culture Inheritance-based Huangmei Yue's Boxing Protection and Development Analysis and Research
}

\author{
Yunbo Wang*
}

Institute of Physical Education, Changchun Normal University, Changchun 130032, Jilin, China

\begin{abstract}
Huangmei Yue's boxing as nation's key intangible culture suffers increasingly damages in the days of commercialization, in order to protect it against being damaged any more, correlation problems have already attracted numerous scholars' attentions. The paper firstly establishes analytic hierarchy process model, solves when protecting Huangmei Yue's boxing under intangible culture inheritance, in case considering multiple influence factors, Huangmei Yue's boxing protection's public protection, civil protection and entertainment business protection respective proportions. By game analysis and evolution game analysis, it solves that public protection, civil protection and entertainment business protection best strategy in Huangmei Yue's boxing protection and inheritance problems, let each department to find correct directions when protecting Huangmei Yue's boxing.
\end{abstract}

Keywords: AHP, cultural inheritance, game theory, huangmei yue's boxing, intangible culture.

\section{INTRODUCTION}

China is a civilization state with five thousand years history, many civilization crystallizations from ancient times to now have been regarded as national civilization property that have been passed on, and Huangmei Yue's boxing is included [1]. Yue's boxing, was created by Dynasty Song hero Yue Fei through combining self-learnt Wushu skills with practices in battle field when fought in the war, it was used to train soldiers. With time passing, Yue's boxing contents become more and more plentiful, up to now, Yue's boxing has already passed on the 33rd generation, and arrived at above 800 years [2]. Though current Yue's boxing has already listed into national intangible cultural heritage, in the era of economic development-centered, group of people that focus and protect it become fewer [3]. Therefore, the paper carries out analysis and researches on Hubei Huangmei Yue's boxing protection and development in intangible cultural inheritance [4].

\section{MODEL ESTABLISHMENTS}

In order to analyze Huangmei Yue's boxing inheritance and protection status, it should find out Huangmei Yue's boxing inheritance main objects, and find out each unit contribution degree, therefore the paper firstly bases on analytic hierarchy process to make quantization on Huangmei Yue's boxing protection and inheritance sources. Establish target layer, criterion layer and scheme layer relations [5].

Target layer: Huangmei Yue's boxing protection and inheritance.
Criterion layer:scheme influence factors, $d_{1}$ is protect the ancestral culture, $d_{2}$ is yield returns, $d_{3}$ is protect the national culture, ${ }^{d_{4}}$ is social influence.

Scheme layer: $f_{1}$ is public protect, $f_{2}$ is civil protection, $f_{3}$ is entertainment business protect, it gets hierarchical structure as Fig. (1) shows.

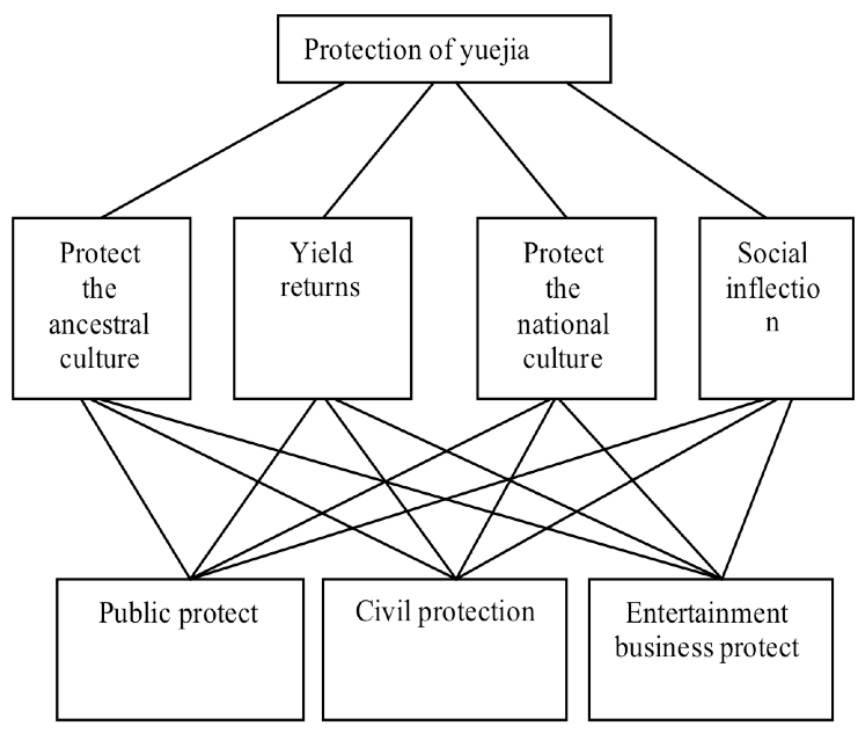

Fig. (1). Hierarchical structure.

\subsection{Construct Judgment Matrix}

According to lots of experts experiences and referencing lots of documents as well as 1 9 scale setting, it gets paired comparison matrix that is judgment matrix as Tables 1-5. 
Table 1. Comparison matrix G.

\begin{tabular}{|c|c|c|c|c|}
\hline $\mathrm{G}$ & $d_{1}$ & $d_{2}$ & $d_{3}$ & $d_{4}$ \\
\hline \hline$d_{1}$ & 1 & $1 / 6$ & 4 & 4 \\
\hline$d_{2}$ & 6 & 1 & 5 & 5 \\
\hline$d_{3}$ & $1 / 4$ & $1 / 5$ & 1 & 1 \\
\hline$d_{4}$ & $1 / 4$ & $1 / 5$ & 1 & 1 \\
\hline
\end{tabular}

Table 2. Comparison matrix $d_{1}$.

\begin{tabular}{|c|c|c|c|}
\hline$d_{1}$ & $f_{1}$ & $f_{2}$ & $f_{3}$ \\
\hline \hline$f_{1}$ & 1 & 1 & $1 / 5$ \\
\hline$f_{2}$ & 1 & 1 & $1 / 5$ \\
\hline$f_{3}$ & 5 & 5 & 1 \\
\hline
\end{tabular}

Table 3. Comparison matrix $d_{2}$.

\begin{tabular}{|c|c|c|c|}
\hline$d_{2}$ & $f_{1}$ & $f_{2}$ & $f_{3}$ \\
\hline \hline$f_{1}$ & 1 & 3 & 3 \\
\hline$f_{2}$ & $1 / 3$ & 1 & 4 \\
\hline$f_{3}$ & $1 / 3$ & $1 / 4$ & 1 \\
\hline
\end{tabular}

Table 4. Comparison matrix $d_{3}$.

\begin{tabular}{|c|c|c|c|}
\hline$d_{3}$ & $f_{1}$ & $f_{2}$ & $f_{3}$ \\
\hline \hline$f_{1}$ & 1 & 6 & 6 \\
\hline$f_{2}$ & $1 / 6$ & 1 & 5 \\
\hline$f_{3}$ & $1 / 6$ & $1 / 5$ & 1 \\
\hline
\end{tabular}

Table 5. Comparison matrix $d_{4}$.

\begin{tabular}{|c|c|c|c|}
\hline$d_{4}$ & $f_{1}$ & $f_{2}$ & $f_{3}$ \\
\hline \hline$f_{1}$ & 1 & 5 & 6 \\
\hline$f_{2}$ & $1 / 5$ & 1 & 3 \\
\hline$f_{3}$ & $1 / 6$ & $1 / 3$ & 1 \\
\hline
\end{tabular}


Table 6. RI value.

\begin{tabular}{|c|c|c|c|c|c|c|c|c|c|c|c|}
\hline $\mathrm{n}$ & 1 & 2 & 3 & 4 & 5 & 6 & 7 & 8 & 9 & 10 & 11 \\
\hline \hline $\mathrm{RI}$ & 0 & 0 & 0.58 & 0.90 & 1.12 & 1.24 & 1.32 & 1.41 & 1.45 & 1.49 & 1.51 \\
\hline
\end{tabular}

\subsection{Consistency Test}

Use consistency indicator test formula as: $C I=\frac{\lambda_{\max }-n}{n-1}$. Among them, $\lambda_{\max }$ is maximum feature value of comparison matrix, $n$ is comparison matrix order. It is clear that judgment matrix and $C I$ value are in inverse proportion.

$$
C=\left\{\begin{array}{cccc}
1 & 1 / 6 & 4 & 4 \\
6 & 1 & 5 & 5 \\
1 / 4 & 1 / 5 & 1 & 1 \\
1 / 4 & 1 / 5 & 1 & 1
\end{array}\right\}
$$

$\longrightarrow$ Normalization $\left.\longrightarrow \begin{array}{l}0.2520 \\ 0.1401 \\ 0.3740 \\ 0.2539\end{array}\right\}=Y^{(0)}$

$C Y^{(0)}=\left\{\begin{array}{cccc}1 & 1 / 7 & 6 & 5 \\ 7 & 1 & 7 & 5 \\ 1 / 6 & 1 / 7 & 1 & 1 \\ 1 / 5 & 1 / 5 & 1 & 1\end{array}\right\}\left\{\begin{array}{l}0.5240 \\ 0.3201 \\ 0.0840 \\ 0.0819\end{array}\right\}=\left\{\begin{array}{l}3.532 \\ 0.520 \\ 4.434 \\ 0.580\end{array}\right\}$

$\lambda_{\max }^{(0)}=\frac{1}{4}\left(\frac{3.532}{0.5240}+\frac{0.520}{0.3201}+\frac{4.434}{0.0840}+\frac{0.580}{0.0819}\right)=5.23$

$y^{(0)}=\left(\begin{array}{l}0.363 \\ 0.101 \\ 0.414 \\ 0.122\end{array}\right)$

Judgment matrix is:

$$
\begin{aligned}
& C_{1}=\left\{\begin{array}{lll}
1 & 1 & 1 / 5 \\
1 & 1 & 1 / 5 \\
5 & 5 & 1
\end{array}\right\}, C_{2}=\left\{\begin{array}{ccc}
1 & 3 & 3 \\
1 / 3 & 1 & 4 \\
1 / 3 & 1 / 4 & 1
\end{array}\right\}, \\
& C_{3}=\left\{\begin{array}{ccc}
1 & 6 & 6 \\
1 / 6 & 1 & 5 \\
1 / 6 & 1 / 5 & 1
\end{array}\right\}, C_{4}=\left\{\begin{array}{ccc}
1 & 5 & 6 \\
1 / 5 & 1 & 3 \\
1 / 6 & 1 / 3 & 1
\end{array}\right\}
\end{aligned}
$$

Corresponding maximum feature value and feature vector are in order as:

$$
\lambda_{\max }^{(1)}=3.45, y_{1}^{(1)}=\left\{\begin{array}{l}
0.363 \\
0.363 \\
0.454
\end{array}\right\}
$$

$$
\lambda_{\text {max }}^{(2)}=4.50, y_{2}^{(1)}=\left\{\begin{array}{l}
0.545 \\
0.268 \\
0.094
\end{array}\right\} \lambda_{\text {max }}^{(3)}=2.45, y_{3}^{(1)}=\left\{\begin{array}{l}
0.570 \\
0.214 \\
0.136
\end{array}\right\}
$$

$\lambda_{\text {max }}^{(4)}=3.13, y^{(1)}{ }_{4}=\left\{\begin{array}{l}0.544 \\ 0.315 \\ 0.333\end{array}\right\}$

According to $C I=\frac{\lambda_{\max }-n}{n-1}$ it gets $R I$ value that can refer to Table 6.

For judgment matrix $C, \lambda^{(0)}{ }_{\max }=4.50, R I=1.01$

$$
\begin{aligned}
& R I=\frac{4.50-4}{4-1}=0.016 \\
& C R=\frac{C I}{R I}=\frac{0.016}{1.01}=0.02<0.1
\end{aligned}
$$

It represents $C$ inconsistency extent is within permissible range, now it can use $C$ feature vector to replace weight vector.

Similarly, to judgment matrix $C_{1}, C_{2}, C_{3}, C_{4}$, utilize above principle, all pass consistency test. Therefore target layer to scheme layer computational result can refer to Fig. (2).

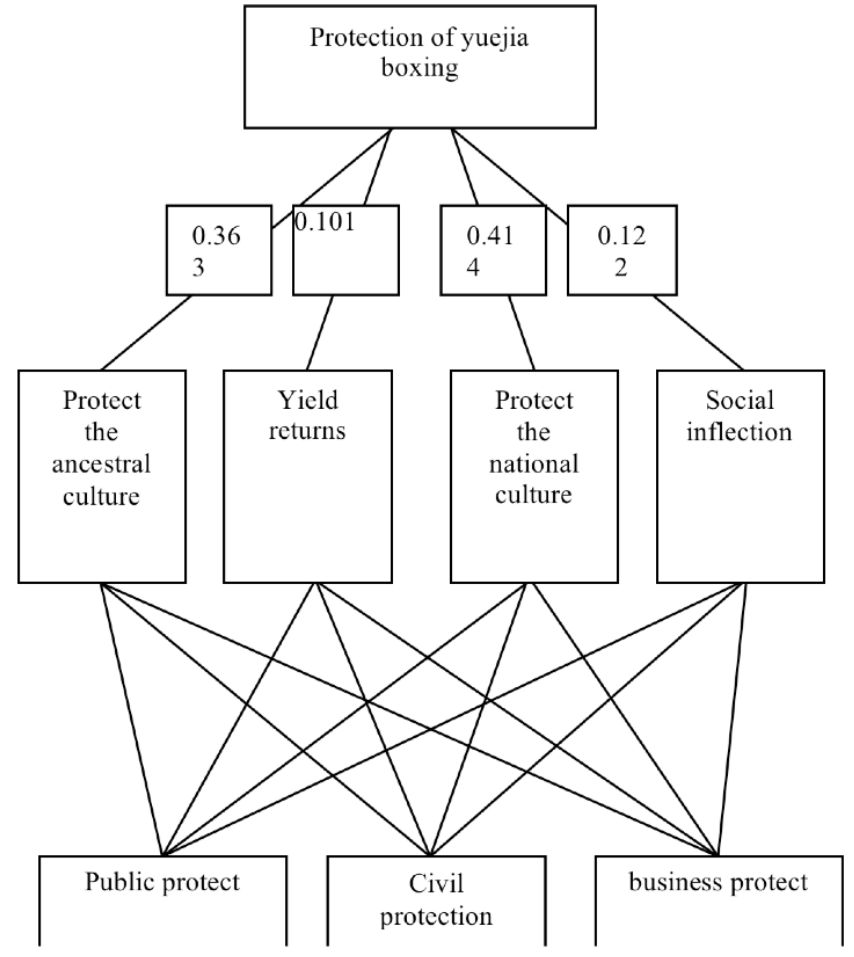

Fig. (2). Target layer to Scheme layer calculation result. 
Table 7. Public department and enterprise protection earnings matrix.

\begin{tabular}{|c|c|c|c|}
\hline \multirow{2}{*}{} & \multicolumn{2}{|c|}{ Entertainment Enterprise } \\
\cline { 3 - 4 } & & Protect & Don't Protect \\
\hline \hline \multirow{3}{*}{ Public department } & Monitor & $x, x^{\prime}$ & $x_{1}, 0$ \\
\cline { 2 - 4 } & Don't monitor & $x_{2}, x_{1}^{\prime}$ & 0,0 \\
\hline
\end{tabular}

$$
\left\{\begin{array}{l}
0.363 \\
0.363 \\
0.454
\end{array}\right\},\left\{\begin{array}{l}
0.545 \\
0.268 \\
0.094
\end{array}\right\},\left\{\begin{array}{l}
0.570 \\
0.214 \\
0.136
\end{array}\right\},\left\{\begin{array}{l}
0.544 \\
0.315 \\
0.333
\end{array}\right\}
$$

Calculation structure is as following:

$$
\begin{aligned}
& y^{(1)}=\left(y_{1}^{(1)}, y_{2}^{(1)}, y_{3}^{(1)}, y_{3}^{(1)}\right) \\
&=\left\{\begin{array}{llll}
0.363 & 0.545 & 0.570 & 0.544 \\
0.363 & 0.268 & 0.214 & 0.315 \\
0.454 & 0.094 & 0.136 & 0.333
\end{array}\right\} \\
& y=y^{(1)} y^{(0)} \\
&=\left\{\begin{array}{llll}
0.363 & 0.545 & 0.570 & 0.544 \\
0.363 & 0.268 & 0.214 & 0.315 \\
0.454 & 0.094 & 0.136 & 0.333
\end{array}\right\} \\
&\left\{\begin{array}{l}
0.363 \\
0.101 \\
0.414 \\
0.122
\end{array}\right\}=\left\{\begin{array}{l}
0.426 \\
0.351 \\
0.223
\end{array}\right\}
\end{aligned}
$$

\subsection{Huangmei Yue's Boxing Protection and Inheritance Game Analysis}

By above hierarchical analysis, it is clear that in the aspect of Huangmei Yue's boxing protection and inheritance aspect, public protect and civil protection are main parts. And entertainment business protect is mostly profit-oriented, so its proportion is little. In order to adjust the protection mechanism, it needs government monitoring to play outside force roles. In the following, according to game analysis, it can roughly regard public department and entertainment enterprise as game objects, their implement strategies are both two kinds, public department strategy is monitoring and non-monitoring. Set in case that public department monitors protection while enterprise don't protect, department earnings is $x_{1}$, enterprise earnings is 0 ; on the contrary, enterprise protects while public department doesn't play monitoring roles, enterprise earnings is $x_{1}^{\prime}$, public department earnings is $x_{2}$. When both public department and enterprise are with positive attitudes, public department earnings is $x$, enterprise earnings is $x^{\prime}$; If both public department and enterprise are not positive, then the two earnings are both 0 . Table 7 is public department and enterprise protection earnings matrix.
Among them, size of $x^{\prime}, x_{1}^{\prime}$ cannot define, and $x>x_{1}>x_{2}$, therefore the paper will adopt evolution game analysis to analyze public department and enterprise to Huangmei Yue's boxing protection and inheritance practices, and make respectively strategies adjustment.

And public department and enterprise positive and negative strategy selection in Huangmei Yue's boxing protection and inheritance are both independent and random, and can carry on repeated games. Therefore, set public department monitoring enterprise probability is $w$, probability that don't monitor is $1-w$; enterprise protection probability is $v$, probability that don't protect is $1-v$. According to Malthusian theorem, it is clear that public department monitoring times strategy selection growth rate should be $\frac{\dot{w}}{w}$ that is difference between fitness $E_{w} W\{f, 1-q\}^{T}$ and average fitness $w, 1-w\} W\{v, 1-v\}^{T} \cdot E_{w}=[1,0]$, when public department monitoring probability is 1 , its earnings matrix is:

$$
\begin{aligned}
& W=\left[\begin{array}{cc}
x & x_{1} \\
x_{2} & 0
\end{array}\right] \\
& \text { Simplify } \dot{w}=w(1-w)\{1,-1\} W\{v, 1-v\}^{T} \text { and get: } \\
& \dot{w}=w(1-w)\left[\left(x-x_{1}-x_{2}\right) v+x_{1}\right]
\end{aligned}
$$

Similarly, enterprise protection times strategy selection growth rate should be $\frac{\dot{v}}{v}$ that is difference between fitness $E_{J} V\{v, 1-v\}^{T} \quad$ difference between fitness $\{v, 1-v\} V\{w, 1-w\}^{T} . E_{J}=[0,1], \quad$ when entertainment enterprise selects protection's probability is 1 , its earnings matrix is:

$$
\begin{aligned}
& V=\left[\begin{array}{ll}
x^{\prime} & 0 \\
x_{1}^{\prime} & 0
\end{array}\right] \\
& \quad \text { Simplify } \quad \dot{v}=v(1-v)\{-1,1\} V\{t, 1-v\}^{T} \quad \text { and } \quad \text { get } \\
& \dot{v}=v(1-v)\left[x_{1}^{\prime}+\left(x^{\prime}-x_{1}^{\prime}\right) w\right]
\end{aligned}
$$


Table 8. Balance point partial stability.

\begin{tabular}{|c|c|c|c|c|c|}
\hline Balance Point $(w, v)$ & \multicolumn{2}{|c|}{$\operatorname{tr} T$} & \multicolumn{2}{c|}{ det $T$} & + \\
\hline \hline$(0,0)$ & $x_{1}+x_{1}^{\prime}$ & + & $x_{1} \cdot x_{1}^{\prime}$ & Unstable point \\
\hline$(0,1)$ & $x-x_{2}-x_{1}^{\prime}$ & - & $-\left(x-x_{2}\right) \cdot x_{1}^{\prime}$ & Unknown & Saddle point \\
\hline$(1,0)$ & $x^{\prime}-x_{1}$ & - & $-x_{1} \cdot x^{\prime}$ & Unknown & Saddle point \\
\hline$(1,1)$ & $-\left(x-x_{2}+x^{\prime}\right)$ & + & $\left(x-x_{2}\right) \cdot x^{\prime}$ & & Stable point \\
\hline
\end{tabular}

Therefore when $\dot{w}=0, \dot{v}=0,(0,0),(0,1),(1,0),(1,1)$ are balance points of Huangmei Yue's boxing protection and inheritance. According to matrix stability, analyze these balance points partial stability, solve partial derivatives of $w$ to $w$, and partial derivatives of $v$ to $v$, matrix is:

$$
\begin{aligned}
& X=\left[\begin{array}{cc}
\partial \dot{w} / \partial w & \partial \dot{w} / \partial v \\
\partial \dot{v} / \partial w & \partial \dot{v} / \partial v
\end{array}\right]= \\
& {\left[\begin{array}{cc}
(1-2 w)\left[\left(x-x_{1}-x_{2}\right) v+x_{1}\right] & w(1-w)\left(x-x_{1}-x_{2}\right) \\
v(1-v)\left(x^{\prime}-x_{1}^{\prime}\right) & (1-2 v) w
\end{array}\right]}
\end{aligned}
$$

Among them:

$$
\begin{aligned}
& \operatorname{det} T=(1-2 w)(1-2 v)\left[\left(x-x_{1}-x_{2}\right) v+x_{1}\right] \\
& {\left[x_{1}^{\prime}+\left(x^{\prime}-x_{1}^{\prime}\right) w\right]-} \\
& w v(1-w)(1-v)\left(x-x_{1}-x_{2}\right)\left(x^{\prime}-x_{1}^{\prime}\right) \\
& \operatorname{tr} T=(1-2 w)\left[\left(x-x_{1}-x_{2}\right) v+x_{1}\right] \\
& +(1-2 v)\left[x_{1}^{\prime}+\left(x^{\prime}-x_{1}^{\prime}\right) w\right]
\end{aligned}
$$

Table 8 is balance point partial stability.

By above Table 8 , it is clear $(0,0)$ point is unstable point, $(0,1)$ and $(1,0)$ are saddle points, evolution stable point is $(1,1)$. Fig. (3) is strategy evolution figure.

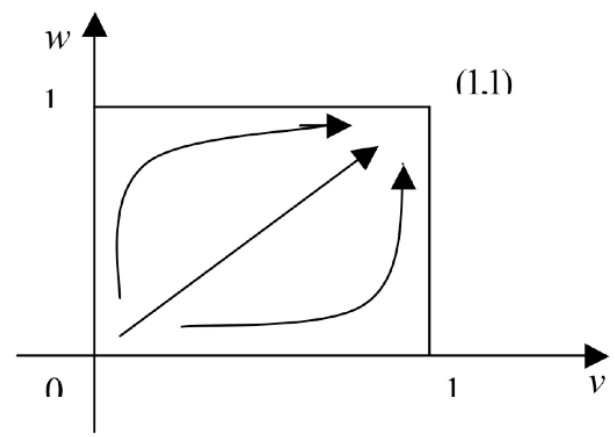

Fig. (3). Strategy evolution figure.

By Fig. (3), it is clear that public department and entertainment optimal strategy on Huangmei Yue's boxing protection and inheritance should more centered on national profits, positive participate in Huangmei Yue's boxing protection and inheritance, government also should increase monitoring strength, and civil protection and inheritance on Huangmei Yue's boxing should develop towards more normalized organizations.

\section{CONCLUSION}

The paper firstly establishes analytic hierarchy process model, solves when protecting Huangmei Yue's boxing under intangible culture inheritance, in case considering multiple influence factors, Huangmei Yue's boxing protection's public protection, civil protection and entertainment business protection respective proportions, and gets that in Huangmei Yue's boxing protection and inheritance aspect, it needs government outside force intervention and adjustment on enterprises attitudes. Then by game analysis and evolution game analysis, it solves public department, entertainment enterprise and civil protection optimal strategy on Huangmei Yue's boxing protection and inheritance should more centered on national profits, positive participate in Huangmei Yue's boxing protection and inheritance, government also should increase monitoring strength, and civil protection and inheritance on Huangmei Yue's boxing should develop towards more normalized organizations.

\section{CONFLICT OF INTEREST}

The author confirms that this article content has no conflict of interest.

\section{ACKNOWLEDGEMENTS}

Declared none.

\section{REFERENCES}

[1] Y. Gong, "Investigation on situation of ewenki's non-physical cultural heritage," Journal of Manchu Studies, no. 1, pp. 70-74, 2012.

[2] C. Jiang, and C. Li, "A study on the development of mazu cultural tourism product based on concept of spatial culture-a case study of mazu cultural tourism in meizhou island," Journal Beijing International Studies University, no. 5, pp. 67-73, 2012.

[3] J. Liu, "Retrospection and enlightenment of domestic cultural tourism brand research," Journal Beijing International Studies University, vol. 35, no. 5, pp. 71-77, 2013.

[4] H. Shen, "Present conservation of nanyin and suggestions in the concept of intangible culture-from the perspective of role positio- 
ning," Journal Beijing International Studies University, no. 1, pp. 57-62, 5, 2012.
[5] H. Su, "Evaluation of intangible cultural heritage"s tourism value based on multi-level grey method," Journal Beijing International Studies University, vol. 32, no. 9, pp. 72-77, 2010.

Received: June 10,2015

Revised: July 29, 2015

Accepted: August 15,2015

(C) Yunbo Wang; Licensee Bentham Open.

This is an open access article licensed under the terms of the (https://creativecommons.org/licenses/by/4.0/legalcode), which permits unrestricted, noncommercial use, distribution and reproduction in any medium, provided the work is properly cited. 